\title{
Specificity of MicroRNA Detection on a Power-free Microfluidic Chip with Laminar Flow-assisted Dendritic Amplification
}

\author{
Kazuki Hasegawa, $* * *$ Rina Negishi, $* * * *$ Mutsuyoshi Matsumoto,** Masafumi Yohda,*** \\ Kazuo HosoKawa, ${ }^{* \dagger}$ and Mizuo MaEDA* \\ *Bioengineering Laboratory, RIKEN, 2-1 Hirosawa, Wako, Saitama 351-0198, Japan \\ **Department of Materials Science and Technology, Tokyo University of Science, 6-3-1 Niijuku, Katsushika, \\ Tokyo 125-8585, Japan \\ ***Department of Biotechnology and Life Science, Tokyo University of Agriculture and Technology, \\ 2-24-16 Naka-cho, Koganei, Tokyo 184-8588, Japan
}

\begin{abstract}
MicroRNAs (miRNAs) are attracting considerable attention as potential biomarkers for the early diagnosis of cancer. We have been developing a detection method for miRNAs on a microfluidic chip with external-power-free fluid pumping and enzyme-free amplification. The assay is completed within $20 \mathrm{~min}$. Here, we describe the specificity of this miRNA detection method. First, the specificity against mismatched sequences was investigated. The nonspecific detection of a 2-nucleotide mismatched sequence was negligible, while that of a 1-nucleotide mismatched sequence was observed to a reasonable extent. Next, the disturbance in mature miRNA detection by existence of its precursor miRNA was evaluated. One precursor miRNA out of four tested showed significant nonspecific responses at $1 \mathrm{nM}$ or higher concentrations. However, those responses were much lower than that of the target mature miRNA at $0.1 \mathrm{nM}$. Finally, we tried to detect three endogenous miRNAs, which are known to be potential cancer biomarkers, in human leucocyte total RNA. The measured concentraions of these miRNAs agreed well with those obtained by quantitative reverse transcription polymerase chain reaction. These results indicate that the on-chip miRNA detection method has good specificity, which is promising for applications to real biological samples.
\end{abstract}

Keywords MicroRNA, detection, power-free microfluidic chip, LFDA, qRT-PCR

(Received July 14, 2016; Accepted September 5, 2016; Published February 10, 2017)

\section{Introduction}

A microRNA (miRNA) is a single-stranded, non-coding RNA with a typical length of $\sim 22$ nucleotides (nt). MiRNAs with various sequences are found in plant and animal cells, and regulate gene expression by binding to their specific target messenger RNAs. The human genome encodes more than 2500 miRNAs, which are estimated to regulate the expression of more than $60 \%$ of all human genes. ${ }^{1}$ Since the first discovery of miRNA in 1993, the importance of the miRNA study has been steadily growing. Especially, several miRNAs have been identified as being key players in the genesis and progression of cancers, ${ }^{2,3}$ which are basically caused by some abnormality in gene expression. Some miRNAs are found in human body fluids such as peripheral blood in a very stable manner. ${ }^{4-7}$ These miRNAs are expected to be potential biomarkers for the early diagnosis of cancer. ${ }^{8-12}$

Conventional methods for miRNA detection and quantification, such as quantitative reverse transcription polymerase chain reaction (qRT-PCR), next-generation sequencing, and microarrays, have their own strengths and drawbacks. ${ }^{13}$

K. Hasegawa and R. Negishi equally contributed to this work.

$\dagger$ To whom correspondence should be addressed.

E-mail: k-hoso@riken.go.jp
Generally, these methods require a long assay time and expensive instruments. Therefore, they are not ideal for diagnostic purposes at the point of care or in resource-poor settings. To overcome these problems, various biosensing devices for rapid and inexpensive miRNA detection have been being developed. ${ }^{14,15}$ For example, Ueno and Funatsu proposed a "ligase-assisted sandwich hybridization" method for miRNA detection on a glass substrate. ${ }^{16}$ Ligation on solid surfaces has also been employed by other research groups. ${ }^{17,18}$ Despite the excellent sensitivity and specificity, the involvement of ligase or other enzymes is potentially a limiting factor in terms of the assay time and cost.

We have been developing a microfluidic chip for rapid and inexpensive miRNA detection. ${ }^{19-21}$ Our method has two distinct features. First, the microfluidic chip requires no external power source for fluid pumping, because the pumping is achieved by degassed poly(dimethylsiloxane) (PDMS), which is widely employed as a microfluidic chip material. ${ }^{22}$ Second, the detection protocol involves no enzymatic reaction, because the fluorescence signal on the microfluidic chip is amplified with our original, enzyme-free technology: laminar flow-assisted dendritic amplification (LFDA). ${ }^{23,24}$ So far, we have reported that miR-21 - a potential cancer biomarker ${ }^{10,11}$ — can be detected within 20 min. ${ }^{20}$ The limit of detection (LOD) was $0.5 \mathrm{pM}$ with a sample volume of only $0.5 \mu \mathrm{L}$. We have also reported that our method can easily be expanded to the simultaneous triplex 
Table 1 Oligomeric RNA sequences used in this work

\begin{tabular}{|c|c|}
\hline Symbol & Sequence ${ }^{\mathrm{a}, \mathrm{b}, \mathrm{c}, \mathrm{d}, \mathrm{e}}$ \\
\hline miR-204 & UUCCCUUUGUC AUCCUAUGCCU \\
\hline miR-211 & UUCCCUUUGUC AUCCUUCGCCU \\
\hline mmu-miR-211 & UUCCCUUUGUC AUCCUUUGCCU \\
\hline miR-16 & UAGCAGCAC GUAAAUAUUGGCG \\
\hline miR-21 & UAGCUUAUCAG ACUGAUGUUGA \\
\hline $\operatorname{miR}-451$ & AAACCGUUAC CAUUACUGAGUU \\
\hline $\operatorname{miR}-500$ & AUGCACCUGG GCAAGGAUUCUG \\
\hline miR-223 & UGUCAGUUUGU CAAAUACCCCA \\
\hline cel-miR-39 & UCACCGGGU GUAAAUCAGCUUG \\
\hline pre-miR-16 & $\begin{array}{l}\text { GUCAGCAGUGCCUUAGCAGCACGUAAAUAUUGGCGUUAAGAUUCUA } \\
\text { AAAUUAUCUCCAGUAUUAACUGUGCUGCUGAAGUAAGGUUGAC }\end{array}$ \\
\hline pre-miR-21 & $\begin{array}{l}\text { UGUCGGGUAGCUUAUCAGACUGAUGUUGACUGUUGAAUCUCAUGGC } \\
\text { AACACCAGUCGAUGGGCUGUCUGACA }\end{array}$ \\
\hline pre-miR-451 & $\begin{array}{l}\text { CUUGGGAAUGGCAAGGAAACCGUUACCAUUACUGAGUUUAGUAAUG } \\
\text { GUAAUGGUUCUCUUGCUAUACCCAGA }\end{array}$ \\
\hline pre-miR-500 & $\begin{array}{l}\text { GCUCCCCCUCUCUAAUCCUUGCUACCUGGGUGAGAGUGCUGUCUG } \\
\text { AAUGCAAUGCACCUGGGCAAGGAUUCUGAGAGCGAGAGC }\end{array}$ \\
\hline
\end{tabular}

a. Written from $5^{\prime}$ to $3^{\prime}$. b. Solid underlines indicate DP-binding sequences. c. Dashed underlines indicate CP-binding sequences. d. Bold letters indicate mismatched bases. e. Italic letters in pre-miRNA sequences indicate mature miRNA sequences.

detection of miR-16, miR-21, and miR-500..$^{21}$ These previous studies were carried out with artificial samples containing only purified target miRNAs. Although the latter paper $^{21}$ revealed that our method produced no cross-reaction between those totally different sequences, the detection specificity of our method has been largely unexplored. A real biological sample usually contains a large excess of off-target RNAs. Especially, the detection of a target miRNA might be disturbed by homologous miRNAs, which sometimes differ by only single base, or by the target's precursor RNAs, which contains the mature target sequence as its partial sequence. ${ }^{25}$

In this paper, we describe the results of our study concerning the specificity of miRNA detection on the power-free microfluidic chip with LFDA. We focused on three issues. First, the specificity against mismatched sequences was studied. We chose three mature miRNA sequences with 1- or 2-nt differences. Using a single type of microfluidic chip matching one of those miRNAs, the responses to the mismatched miRNAs were evaluated. Second, the specificity against precursor RNAs was studied. For four mature miRNA sequences, the shortest types of precursor RNAs (pre-miRNAs) were adopted. The responses of the microfluidic chips matching the mature miRNAs to their corresponding pre-miRNAs were evaluated. Finally, the detection of endogenous miRNAs in a total RNA sample derived from human leucocytes was carried out. The results were compared with measurement by qRT-PCR.

\section{Experimental}

\section{Oligonucleotides}

Table 1 lists the oligomeric RNA sequences used in this work. The mature miRNA sequences (22 nt) were obtained from Greiner. The pre-miRNAs $(72-89 \mathrm{nt})$ were obtained from Integrated DNA Technologies. Sequences of capture probe (CP) DNAs and detection probe (DP) DNAs are listed in Table S1 in the Supporting Information (SI). They were both obtained from Eurofins Genomics. The $5^{\prime}$ end of each CP was aminated, while the $3^{\prime}$ end of each DP was biotinylated.

\section{Microfluidic chip}

The design of the microfluidic chip was described elsewhere. ${ }^{20}$ Briefly, it consisted of a $30 \times 25 \times 2 \mathrm{~mm}$ PDMS part and a $76 \times 26 \times 1 \mathrm{~mm}$ glass substrate. A piece of adhesive tape was used for the power-free pumping technique. ${ }^{22}$ A straight-line pattern (100 $\mu \mathrm{m}$ wide) of CP was made on the glass substrate, which was then covered with the PDMS part having microchannels $(100 \mu \mathrm{m}$ wide and $25 \mu \mathrm{m}$ deep), so that the $\mathrm{CP}$ pattern and the microchannels became orthogonal to each other. A single assay was carried out with a pair of Y-shaped microchannels sharing one inlet ("center inlet" in Fig. 1). The left Y-channel was used for the miRNA sample, while the right one was for a blank solution as a reference.

The PDMS part was fabricated as described elsewhere. ${ }^{22}$ Briefly, a negative master for the molding of PDMS was fabricated on a silicon wafer with ultrathick photoresist (SU-8 25 , MicroChem). The surface of the master was passivated by exposure to $\mathrm{CHF}_{3}$ plasma in a plasma etcher (RIE-10NR, Samco International). Prepolymer of PDMS (Sylgard 184, Dow Corning) was cast onto the master with a frame for holding the prepolymer. The cured PDMS part was peeled off from the master, and thorough-holes for the waste reservoirs were punched using a metal pipe. To make the inlet openings, the PDMS part was cut with a knife.

The CP was immobilized onto the glass surface as described elsewhere. ${ }^{20}$ Briefly, $1 \%$ glutaraldehyde in carbonate buffer was reacted with an aminated glass slide (SD00011, Matsunami Glass) at $37^{\circ} \mathrm{C}$ for $2 \mathrm{~h}$. A PDMS part having a straight microchannel was attached onto the glass surface. The CP $(100 \mu \mathrm{M})$ in ultrapure water was injected into the microchannel and incubated at $37^{\circ} \mathrm{C}$ for $1 \mathrm{~h}$. The PDMS part was detached from the glass surface in a stopping buffer $(25 \mathrm{mM}$ Tris, $0.05 \%$ Tween 20), and the glass substrate was washed by ultrasonication in the stopping buffer, followed by ultrapure water. The PDMS part having the Y-shaped microchannels was attached onto the glass surface. The PDMS-glass microfluidic chip was degassed in a vacuum chamber at $10 \mathrm{kPa}$ for $1 \mathrm{~h}$ for power-free pumping.

\section{On-chip miRNA detection}

The protocol for on-chip miRNA detection is illustrated in Fig. 1 (lower left). It consisted of three steps. All of the 


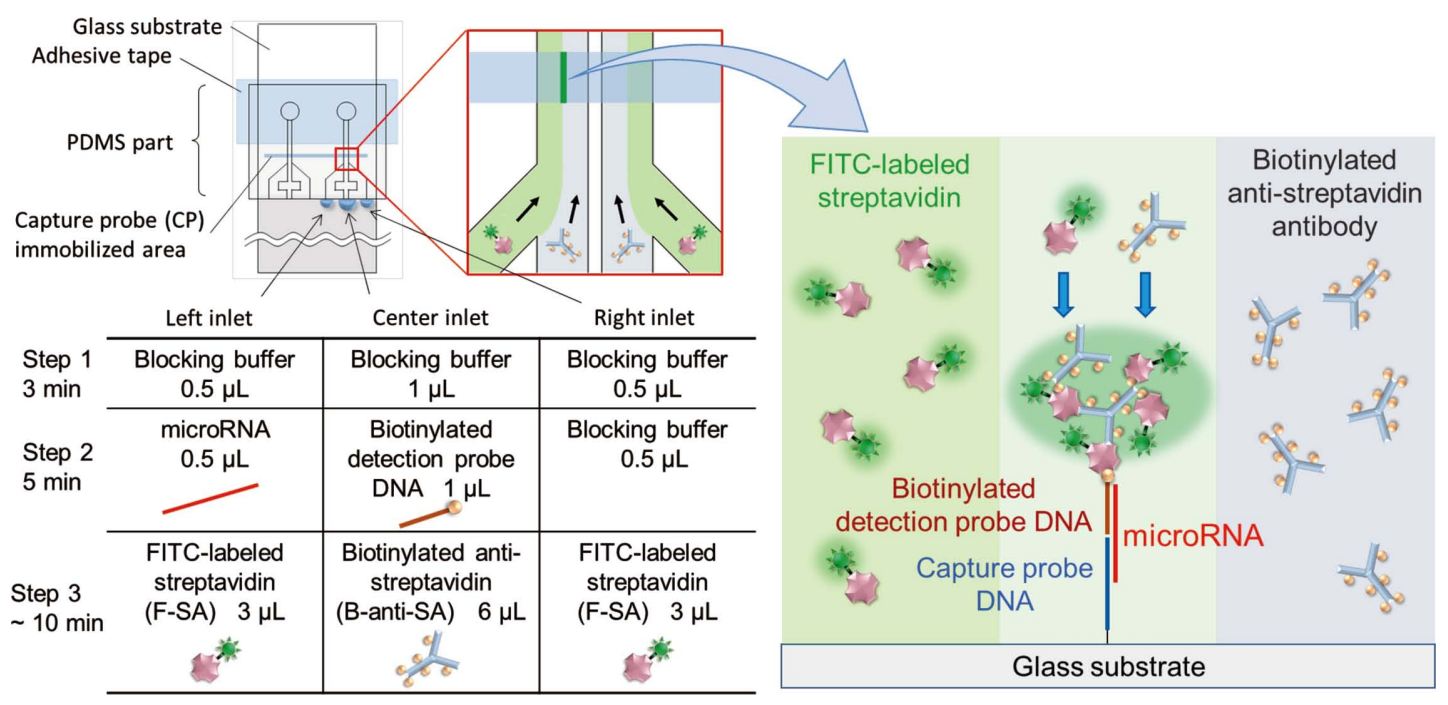

Fig. 1 Schematic representation of the on-chip detection method for miRNA.

solutions were injected with the power-free pumping technique. ${ }^{22}$ In each step, three aliquots were injected almost simultaneously (<40 s). (Step 1) A blocking buffer (1\% Roche Blocking Reagent, $0.02 \%$ (w/v) SDS, $0.05 \%$ Tween 20, and 5× SSC) was injected from all of the inlets. The injected volumes were $0.5 \mu \mathrm{L}$ (left and right) and $1 \mu \mathrm{L}$ (center). This blocking buffer was used for dilution of all the solutions in the rest of this protocol. (Step 2) $0.5 \mu \mathrm{L}$ of miRNA sample solution was injected from the left inlet, while the blocking buffer was injected from the right inlet as a blank reference. $1 \mu \mathrm{L}$ of $1 \mu \mathrm{M}$ DP was injected from the center inlet. (Step 3) $3 \mu \mathrm{L}$ of $5 \mathrm{ng} / \mu \mathrm{L}$ FITC-labeled streptavidin (F-SA) was injected from the left and right inlets, while $6 \mu \mathrm{L}$ of $10 \mathrm{ng} / \mu \mathrm{L}$ biotinylated anti-streptavidin antibody (B-anti-SA) was injected from the center inlet. Fluorescence images were taken every $1 \mathrm{~min}$ on an inverted fluorescence microscope (TE2000-U, Nikon) with a 10x objective lens and a cooled charge-coupled device camera (CoolSNAP HQ2, Photometrics). The images were analyzed using ImageJ software (National Institutes of Health).

Prior to the detection experiment, only pre-miRNAs were thermally annealed so that the RNA molecules formed the proper secondary structures. Specifically, the pre-miRNA solution was heated at $90^{\circ} \mathrm{C}$ for 10 min with a thermal cycler (Arktik, Thermo Fisher Scientific), then gradually cooled down to $4^{\circ} \mathrm{C}$ in $1 \mathrm{~h}$.

\section{$q R T-P C R$}

Total RNA from human leucocytes was purchased from Clontech (cat. \#636592). Reverse transcription (RT) and PCR were carried out using miRCURY LNA Universal RT microRNA PCR kit (Exiqon). The RT mixture consisted of $2 \mu \mathrm{L}$ of $5 \mathrm{ng} / \mu \mathrm{L}$ total RNA, $1 \mu \mathrm{L}$ of Enzyme Mix, $2 \mu \mathrm{L}$ of $5 \times$ Reaction Buffer, and $5 \mu \mathrm{L}$ of nuclease-free water. The reaction was carried out at $42^{\circ} \mathrm{C}$ for $60 \mathrm{~min}$, and stopped by heating at $95^{\circ} \mathrm{C}$ for $5 \mathrm{~min}$ on the thermal cycler. The cDNA product was stored at $4{ }^{\circ} \mathrm{C}$. Prior to real-time PCR, the cDNA product was diluted 76-fold with ultrapure water. The PCR mixture consisted of $3.8 \mu \mathrm{L}$ of the diluted cDNA, $0.2 \mu \mathrm{L}$ of ROX Passive Reference, $1 \mu \mathrm{L}$ of PCR Primer Mix, and $5 \mu \mathrm{L}$ of PCR Master Mix. Simple calculation reveals that this PCR mixture contained cDNA derived from $0.05 \mathrm{ng}$ of the total RNA. For calibration purpose, standard samples were prepared by replacing the total RNA in the RT mixture described above with $2 \mu \mathrm{L}$ of $10^{5}-10^{9}$ copies $/ \mu \mathrm{L}$ synthesized miRNA (Table 1) dissolved in $5 \mathrm{ng} / \mu \mathrm{L}$ MS2 RNA (Roche) as carrier RNA. Calibration was carried out using PCR mixtures containing the standard cDNA samples. Real-time PCR was carried out on 7900HT Fast Real Time PCR System (Applied Biosystems) under the following temperature conditions: starting at $95^{\circ} \mathrm{C}$ for $10 \mathrm{~min}$ and then 40 cycles of $95^{\circ} \mathrm{C}$ for $10 \mathrm{~s}$ and $60^{\circ} \mathrm{C}$ for $1 \mathrm{~min}$ with a ramp rate of $1.6^{\circ} \mathrm{C} / \mathrm{s}$. The data were analyzed with SDS Software v2.4 (Applied Biosystems).

\section{Results and Discussion}

The detection strategy for miRNA on the power-free microfluidic chip with LFDA is illustrated in Fig. 1 (right). The target miRNA is sandwiched by the immobilized CP DNA and the biotinylated DP DNA. In the next step, LFDA is carried out by injecting F-SA and B-anti-SA from different inlets. The two kinds of molecules are continuously supplied from the two laminar streams to the biotin moiety of the DP DNA molecules, and aggregate together there. As a result, the fluorescence signal is amplified by accumulation of the F-SA molecules. In the neighboring microchannel, a blank solution is introduced as a reference.

A typical fluorescence image of the microchannels during LFDA is shown in Fig. 2(a). In the sample channel, strong fluorescence was observed at the expected location where $\mathrm{CP}$, target miRNA, DP, F-SA, and B-anti-SA are considered to have existed. In contrast, only faint fluorescence was observed at the corresponding position in the blank reference channel. In fact, this image shows the very beginning of nonspecific LFDA in the blank channel, as evidenced by the time course shown in Fig. 2(b). When the image was taken (6 min), the time course of the blank was steeply bent upward, indicating the beginning of nonspecific LFDA. Every time course plot in this study had a similar bending point. We objectively determined the bending point in the time course plot of the blank reference by calculating the changing rate of the slopes at every point along the plot. We defined the "signal-to-blank ratio" (SBR) as the fluorescence of the sample channel divided by the fluorescence of the blank channel at the bending point (the arrow in Fig. 2(b)). The SBR 
(a)

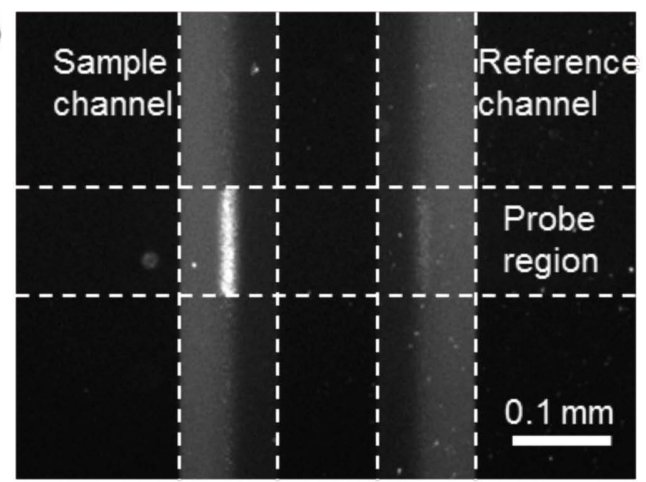

(b)

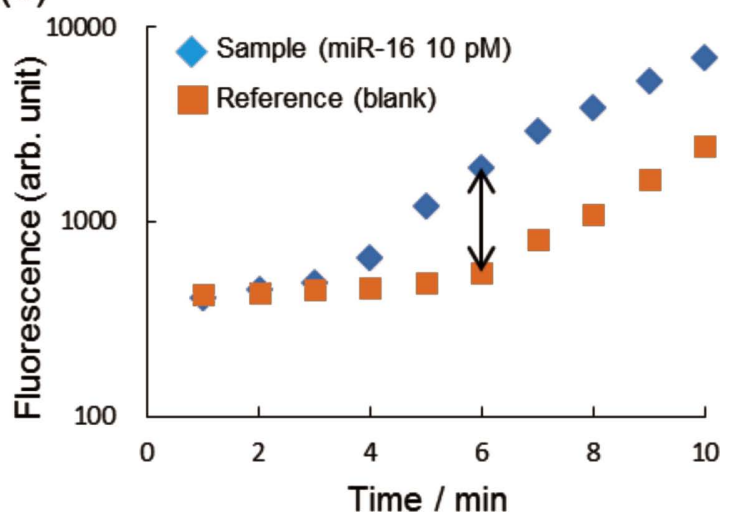

Fig. 2 (a) Typical fluorescence image of the microchannels during LFDA. The target was $10 \mathrm{pM}$ miR-16. The LFDA time was 6 min. (b) Time course of the fluorescence in the sample channel (diamonds) and the reference channel (squares) in the experiment described above. The arrow indicates the signal-to-blank ratio (SBR, see text).

is used for quantifying the results from microfluidic chips throughout this work.

We selected nine mature miRNAs as the targets (Table 1). They are found in human tissues, except for mmu-miR-211 (mouse) and cel-miR-39 (C. elegans). We selected them for different reasons: sequence similarity (miR-204, miR-211, and mmu-miR-211), potential as cancer biomarkers (miR-16, ${ }^{12}$ miR-21, ${ }^{10,11}$ miR-451, ${ }^{12}$ miR-500, ${ }^{8}$ and miR-223 ${ }^{11}$ ), and lack of homology with human RNA (cel-miR-39). For each target sequence, we designed $\mathrm{CP}$ and DP sequences by calculating dissociation constants of CP-target binding and DP-target binding on the "UNAFold" web site. ${ }^{26}$ We determined the CP and DP sequences so that the two dissociation constants became close to each other. The binding sequences to the probes are indicated by underlines in Table 1 , and the probe sequences are explicitly shown in Table S1 in the SI.

First, we investigated the specificity of the on-chip miRNA detection method in terms of the sequence similarity. We fabricated microfluidic chips for the detection of miR-204. Using these chips and the DP which matches miR-204, detection experiments were carried out for miR-204, mmu-miR-211 (1-nt mismatch), and miR-211 (2-nt mismatch) at various concentrations. The resulting time-course plots are shown in Fig. S1 in the SI. The obtained SBR values are plotted in Fig. 3. As expected, miR-204 produced the highest SBR values of these three sequences; these SBR values are positively correlated with the concentrations. In contrast, miR-211 produced SBR values consistently below the $3 \sigma$ line of miR-204 (the definition of the $3 \sigma$ line is described in Fig. 3 legend). This means that

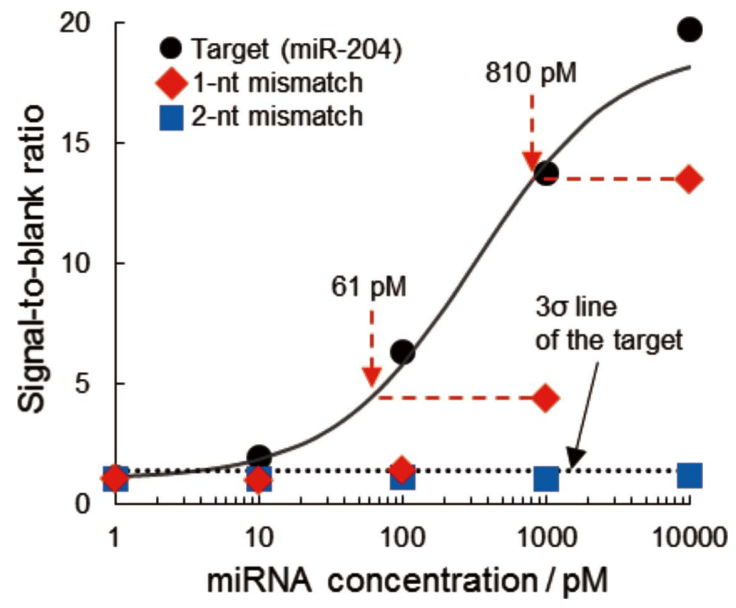

Fig. 3 Calibration plots of miR-204 (target), mmu-miR-211 (1-nt mismatch), and miR-211 (2-nt mismatch) obtained by using the CPDP pair which fully matches miR-204. The solid curve was obtained by least-square fitting of the miR-204 data with the four-parameter logistic function. The " $3 \sigma$ line" was drawn at calculated zero-dose SBR of miR-204 plus 3-times standard deviation of zero-dose measurements $(n=5)$. Specifically, the zero-dose measurements resulted in fluorescence values of $520 \pm 70$ a.u. for the signal and $470 \pm 80$ a.u. for the blank. The SBR value was $1.1 \pm 0.1$.

the responses to miR-211 at $10 \mathrm{nM}$ or lower was not statistically different from those to the blank sample. Therefore, the specificity against the 2-nt mismatch was perfect in this set of experiments. However, this was not the case for the 1-nt mismatch (mmu-miR-211), which produced significant SBR values at very high concentrations (1 and $10 \mathrm{nM})$. The fitting curve allows us to translate the SBR values into concentrations under the assumption that we mistake mmu-miR-211 as miR-204. The results are $6.1 \%(61 \mathrm{pM} / 1 \mathrm{nM})$ and $8.1 \%$ (810 pM/10 nM) of the input mmu-miR-211. The impact of this level of error may depend on the specific application. One possible way to avoid this error would be simultaneous usage of both probe sets for the two similar sequences in a multiplex detection format. ${ }^{21}$ The response from one probe set could be used to correct the response from the other probe set, and vice versa.

Next, we investigated the specificity of mature miRNA detection against its precursor, pre-miRNA. In living cells, miRNAs are known to be produced by cleavage from premiRNAs, which form hairpin (stem-loop) structures with typical lengths of around $70 \mathrm{nt}^{25}$ Because pre-miRNAs naturally includes their mature miRNA sequences, there is a potential risk of interference of pre-miRNAs with the detection of mature miRNAs. To study this effect, we selected four pre-miRNAs from an online database "miRBase", ${ }^{27}$ and tried to detect them on microfluidic chips designed for the detection of their mature counterparts. We first confirmed that these microfluidic chips correctly responded to their authentic targets at $0.1 \mathrm{nM}$ (Fig. 4(a), upper row). The same microfluidic chips were used for detection tests of corresponding pre-miRNAs at 100-fold excess concentration, $10 \mathrm{nM}$. Immediately before these tests, the pre-miRNA solutions were thermally annealed from $90^{\circ} \mathrm{C}$ to $4^{\circ} \mathrm{C}$ in $1 \mathrm{~h}$ so as to ensure the formation of the proper hairpin structures (Fig. 4(b)). As a result, negligible responses were observed with pre-miR-16, -21 , and -451 . However, a significant response was observed with pre-miR-500 (Fig. 4(a), lower row), although this response was much lower than that with mature 
(a)
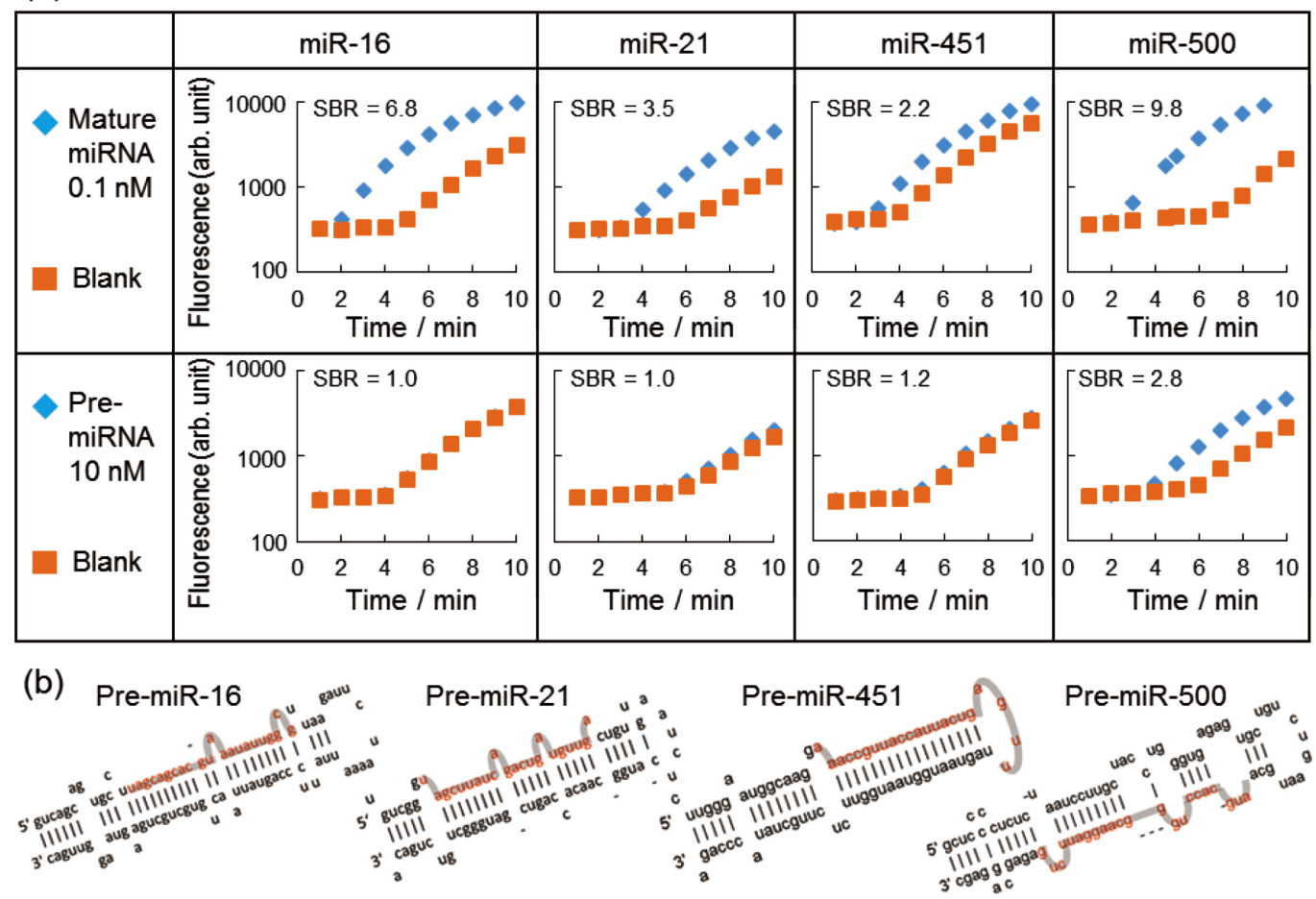

Fig. 4 (a) Time course plots of on-chip detection for mature miRNAs at $0.1 \mathrm{nM}$ (upper row) and nonspecific responses to the pre-miRNAs at $10 \mathrm{nM}$ (lower row). (b) Predicted structures of the premiRNAs. ${ }^{27}$ The shaded parts become the mature miRNAs by cleavage in living cells.

miR-500 at the 100-fold lower concentration. The greater nonspecific response to pre-miR-500 than those to other pre-miRNAs might be attributable to an instability of the mature miRNA part embedded in the hairpin structure (Fig. 4(b), shaded part). In fact, the embedded mature miRNA part in pre-miR-500 has 7 mismatched bases, compared to 2 (premiR-16), 4 (pre-miR-21), and 5 (pre-miR-451) mismatched bases.

The nonspecific response to pre-miR-500 was evident only when its concentration was very high. The response was confirmed to decrease with the decreasing concentration, and to become negligible at $0.1 \mathrm{nM}$ : the same concentration of the mature miRNA experiments (Fig. 5). We also studied the effects of the thermal treatment of pre-miRNAs before the detection experiments. Instead of the gradual cooling described above, we tried sudden cooling on ice. This treatment is considered to inhibit the formation of the proper hairpin structures. As a result, all of the four pre-miRNAs at $10 \mathrm{nM}$ showed significant responses (Fig. S2 in the SI). These data support the hypothesis that the hairpin structure of the pre-miRNA prevents nonspecific binding to probe DNAs. Overall, our detection method is unlikely to be seriously disturbed by precursor miRNAs as long as their concentrations are not extremely high. However, some consideration might be needed concerning thermal treatment of the sample solution before the assay, not to destroy the hairpin structures of the precursors.

Finally, we attempted the detection of endogenous miRNAs from human leucocyte total RNA, which was commercially available. As model targets, we chose miR-16, -451, and -223, because they were reported as potential cancer biomarkers. ${ }^{11,12}$ We also chose cel-miR-39, which has no homology with human RNA. For each miRNA, we made a calibration curve by (a)
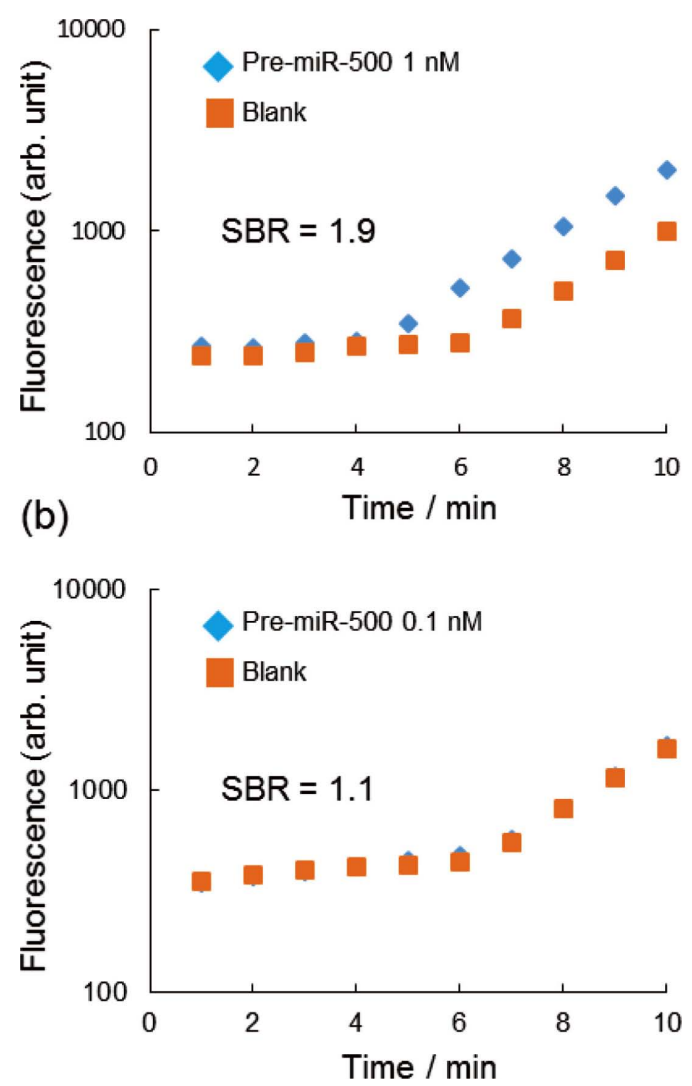

Fig. 5 Time course plots of nonspecific responses to pre-miR-500 at decreasing concentrations, (a) $1 \mathrm{nM}$, and (b) $0.1 \mathrm{nM}$. 


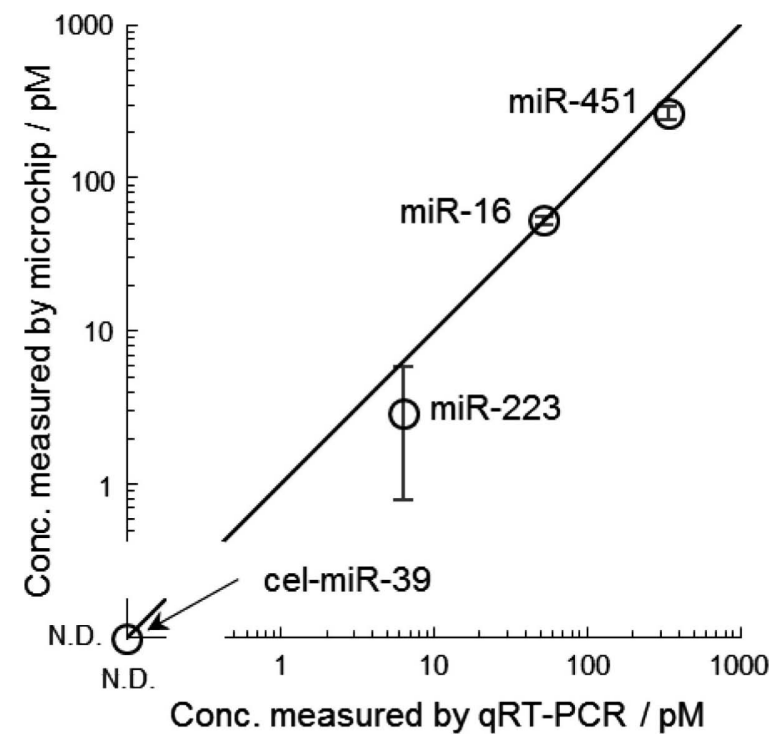

Fig. 6 Correlation between concentrations of miRNAs in $1 \mathrm{ng} / \mu \mathrm{L}$ human leucocyte total RNA measured by microfluidic chips and those measured by qRT-PCR. The error bars were calculated from \pm 1 SD of SBR values $(n=3$, see Fig. S3 in the SI). The solid line represents the ideal relationship, $y=x$. "N.D." by the microfluidic chip means concentration below $0.2 \mathrm{pM}$ : the LOD of cel-miR-39 determined by other experiments. "N.D." by qRT-PCR means Ct greater than 40 .

measuring the SBR values of the synthesized miRNA at various concentrations (Fig. S3 in the SI). In the same way, we measured the SBR values from $1 \mathrm{ng} / \mu \mathrm{L}$ total RNA. The SBR values were translated into concentrations using the calibration curves. The same miRNAs in the total RNA were also measured by qRT-PCR, which primarily produces threshold cycle $(\mathrm{Ct})$ values. We translated the $\mathrm{Ct}$ values into concentrations using the calibration curves shown in Fig. S4 in the SI. The measured values from the two methods are compared in Fig. 6. For miR-451 and miR-16, which were highly expressed in this sample, the two methods showed excellent agreements. The agreement became moderate for miR-223, which was less expressed than the aforementioned miRNAs. Cel-miR-39 was not detected with both of the methods, as expected. These results suggest that the on-chip miRNA detection method has enough specificity for application to a real biological sample, as long as it contains a sufficient amount of the target. For less-abundant targets, an improvement of the sensitivity of the microfluidic chip or preconcentration at the sample preparation step would be desirable.

\section{Conclusions}

The specificity of miRNA detection on the power-free microfluidic chip with LFDA has been investigated. For the 2-nt mismatched sequence, the on-chip detection showed perfect specificity up to $10 \mathrm{nM}$. For the 1-nt mismatched sequence, the microfluidic chip produced some nonspecific response, which may cause an error of less than $10 \%$ in terms of the concentration. Pre-miRNAs were revealed to cause only a slight interference with detection of mature miRNAs, unless the concentration of the pre-miRNAs was extremely high. This inactivity of premiRNAs is probably attributable to their stable secondary structures. The microfluidic chip was successfully applied to the detection of three endogenous miRNAs in human leucocyte total RNA at a concentration of $1 \mathrm{ng} / \mu \mathrm{L}$. The measured concentraions of these miRNAs, ranging from a few picomolar to sub-nanomolar, agreed well with those by qRT-PCR. The assay time was less than $20 \mathrm{~min}$. In the next step of our development, we are planning to tackle two issues: 1) the application of this method to real patient samples, and 2) miniaturization of the optical setup, which is now the bulky and expensive microscope.

\section{Acknowledgements}

This work was suppoerted by KAKENHI (25350581), JST Center of Innovation Program, and RIKEN Junior Research Associate Program.

\section{Supporting Information}

Probe DNA sequences, additional data of the experiments for mismatched miRNAs and pre-miRNAs, and calibration curves for on-chip and qRT-PCR measurements. This material is available free of charge on the Web at http://www.jsac.or.jp/ analsci.

\section{References}

1. R. C. Friedman, K. K. H. Farh, C. B. Burge, and D. P. Bartel, Genome Res., 2009, 19, 92.

2. M. V. Iorio, M. Ferracin, C. G. Liu, A. Veronese, R. Spizzo, S. Sabbioni, E. Magri, M. Pedriali, M. Fabbri, M. Campiglio, S. Menard, J. P. Palazzo, A. Rosenberg, P. Musiani, S. Volinia, I. Nenci, G. A. Calin, P. Querzoli, M. Negrini, and C. M. Croce, Cancer Res., 2005, 65, 7065.

3. G. A. Calin and C. M. Croce, Nat. Rev. Cancer, 2006, 6, 857.

4. P. S. Mitchell, R. K. Parkin, E. M. Kroh, B. R. Fritz, S. K. Wyman, E. L. Pogosova-Agadjanyan, A. Peterson, J. Noteboom, K. C. O'Briant, A. Allen, D. W. Lin, N. Urban, C. W. Drescher, B. S. Knudsen, D. L. Stirewalt, R. Gentleman, R. L. Vessella, P. S. Nelson, D. B. Martin, and M. Tewari, Proc. Natl. Acad. Sci. U. S. A., 2008, 105, 10513.

5. J. A. Weber, D. H. Baxter, S. L. Zhang, D. Y. Huang, K. H. Huang, M. J. Lee, D. J. Galas, and K. Wang, Clin. Chem., 2010, 56, 1733.

6. A. Turchinovich, L. Weiz, A. Langheinz, and B. Burwinkel, Nucleic Acids Res., 2011, 39, 7223.

7. K. Wang, Y. Yuan, J. H. Cho, S. McClarty, D. Baxter, and D. J. Galas, PLoS One, 2012, 7, e41561

8. Y. Yamamoto, N. Kosaka, M. Tanaka, F. Koizumi, Y. Kanai, T. Mizutani, Y. Murakami, M. Kuroda, A. Miyajima, T. Kato, and T. Ochiya, Biomarkers, 2009, 14, 529.

9. N. Kosaka, H. Iguchi, and T. Ochiya, Cancer Sci., 2010, 101, 2087.

10. M. Tsujiura, D. Ichikawa, S. Komatsu, A. Shiozaki, H. Takeshita, T. Kosuga, H. Konishi, R. Morimura, K. Deguchi, H. Fujiwara, K. Okamoto, and E. Otsuji, Brit. J. Cancer, 2010, 102, 1174.

11. J. Zhou, L. Yu, X. Gao, J. Hu, J. P. Wang, Z. Dai, J. F. Wang, Z. Y. Zhang, S. H. Lu, X. W. Huang, Z. Wang, S. J. Qiu, X. Y. Wang, G. H. Yang, H. C. Sun, Z. Y. Tang, Y. Wu, H. G. Zhu, and J. Fan, J. Clin. Oncol., 2011, 29, 4781.

12. C. Zhu, C. Ren, J. Han, Y. Ding, J. Du, N. Dai, J. Dai, H. $\mathrm{Ma}$, Z. Hu, H. Shen, Y. Xu, and G. Jin, Brit. J. Cancer, 
2014, 110, 2291.

13. M. Baker, Nat. Methods, 2010, 7, 687.

14. B. N. Johnson and R. Mutharasan, Analyst, 2014, 139, 1576.

15. R. M. Graybill and R. C. Bailey, Anal. Chem., 2016, 88 , 431.

16. T. Ueno and T. Funatsu, PLoS One, 2014, 9, e90920

17. H. Lee, R. L. Srinivas, A. Gupta, and P. S. Doyle, Angew. Chem., Int. Ed., 2015, 54, 2477.

18. S. Hofmann, Y. W. Huang, P. Paulicka, A. Kappel, H. A Katus, A. Keller, B. Meder, C. F. Stahler, and W. Gumbrecht, Anal. Chem., 2015, 87, 12104.

19. H. Arata, H. Komatsu, A. Han, K. Hosokawa, and M. Maeda, Analyst, 2012, 137, 3234.

20. H. Arata, H. Komatsu, K. Hosokawa, and M. Maeda, PLoS
One, 2012, 7, e48329.

21. R. Ishihara, K. Hasegawa, K. Hosokawa, and M. Maeda, Anal. Sci., 2015, 31, 573.

22. K. Hosokawa, K. Sato, N. Ichikawa, and M. Maeda, Lab Chip, 2004, 4, 181.

23. K. Hosokawa, M. Omata, and M. Maeda, Anal. Chem., 2007, 79, 6000 .

24. K. Hosokawa and M. Maeda, Lab Chip, 2009, 9, 464.

25. R. I. Gregory and R. Shiekhattar, Cancer Res., 2005, 65, 3509.

26. "UNAFold" web site, http://unafold.rna.albany.edu/, accessed July 11, 2016.

27. "miRBase" web site, http://www.mirbase.org/index.shtml, accessed July 11, 2016. 